\title{
Current Driven tri-stable Resistance States in Magnetic Point Contacts
}

\author{
I. K. Yanson ${ }^{1}$, V. V. Fisun ${ }^{1}$, Yu. G. Naidyuk ${ }^{1}$, O. P. Balkashin ${ }^{1}$, \\ L. Yu. Triputen ${ }^{1}$, A. Konovalenko ${ }^{2}$, and V. Korenivski ${ }^{2}$ \\ ${ }^{1}$ B. Verkin Institute for Low Temperature Physics and Engineering, \\ National Academy of Sciences of Ukraine, ${ }^{7} 7$ Lenin ave., 61103, Kharkiv, Ukraine and \\ ${ }^{2}$ Nanostructure Physics, Royal Institute of Technology, 10691, Stockholm, Sweden
}

\begin{abstract}
Point contacts between normal and ferromagnetic metals are investigated using magnetoresistance and transport spectroscopy measurements combined with micromagnetic simulations. Pronounced hysteresis in the point-contact resistance versus both bias current and external magnetic field are observed. It is found that such hysteretic resistance can exhibit, in addition to bi-stable resistance states found in ordinary spin valves, tri-stable resistance states with a middle resistance level. We interpret these observation in terms of surface spin-valve and spin-vortex states, originating from a substantially modified spin structure at the ferromagnetic interface in contact core. We argue that these surface spin states, subject to a weakened exchange interaction, dominate the effects of spin transfer torques on the nanometer scale.
\end{abstract}

PACS numbers:

Spin Transfer Torques (STT) [1, 2] between the conduction electrons and the magnetic lattice in a ferromagnet can cause a rotation of the magnetization when the electron current density is sufficiently high and spinpolarized. The most common geometry for using this effect is a spin-valve with two closely spaced ferromagnetic layers, where one is magnetically hard and acts as the current polarizer, and the other is magnetically soft and can magnetically precess or switch from the action of the polarized current. It has recently been demonstrated that similar in origin magnetization excitations occur for single ferromagnetic films and interfaces [3, 4, 5], where the STT is intra-layer and is mediated by impurity scattering [6]. We have recently shown [7] using ultra thin Co films that STT driven switching can occur in atomically thin spin layers at nonmagnetic/ferromagnetic (N/F) interfaces, which form spin-valve like states with respect to the interior spins, within the same ferromagnetic film. Here we present experimental results indicating that such surface spin states can form stable spin vortices, and the current driven STT switching in the system can involve parallel, anti-parallel, and vortex spin states, yielding three stable resistance states of the ferromagnetic interface.

The STT literature to date [8] has essentially ignored the fact that the spin states at a ferromagnetic interface can have distinctly different properties from those of the interior spins. This fact should be of crucial importance since the STT effect is in nature a surface effect 9]. In the ideal case, universally assumed to be valid in the STT studies, the fundamental characteristics of the magnetic interface - the exchange strength, magnetization magnitude, and anisotropy strength and direction - are assumed to be identical to those in the interior of the ferromagnet. In this case the interface and the interior spins respond as one system to a current driven STT, which is known to be concentrated in an atomi-

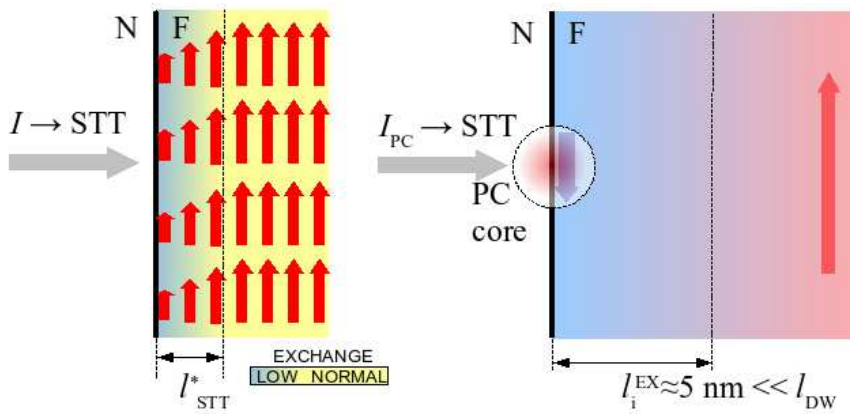

(a)

(b)

FIG. 1: (Color online) a) Schematic of the spin structure at a $\mathrm{N} / \mathrm{F}$ interface where the exchange strength, magnetization magnitude, and anisotropy strength and direction can be significantly different from those in the bulk. The spin angular momentum of the electron current flowing through the interface is transferred to the magnetization of the ferromagnetic layer within an atomically thin layer $\left(l_{\mathrm{STT}}\right)$. b) Illustration of the ferromagnetic length scales in Co on the scale of the our smallest PC exhibiting STT hysteresis. The PC size is an order of magnitude smaller than the characteristic domain wall thickness in Co. The PC core (red) is the region of highest current density, where the STT is strongest.

cally thin layer at the ferromagnetic surface [9]. In the realistic case shown in the Fig. 1(a), the exchange, magnetization, and anisotropy can be significantly different at the interface from those in the bulk. For example, recent surface versus bulk magnetization measurements for Co and $\mathrm{Fe}$ [10, 11] show different anisotropy and coercivity for the surface and interior spins. This should significantly modify the response of the N/F interface to a current driven STT, which we indeed observe [7].

In this paper we study point contacts (PC's) between Co films of different thickness and sharpened wires of normal metal $(\mathrm{Ag}, \mathrm{Cu})$. The films and PCs were prepared 


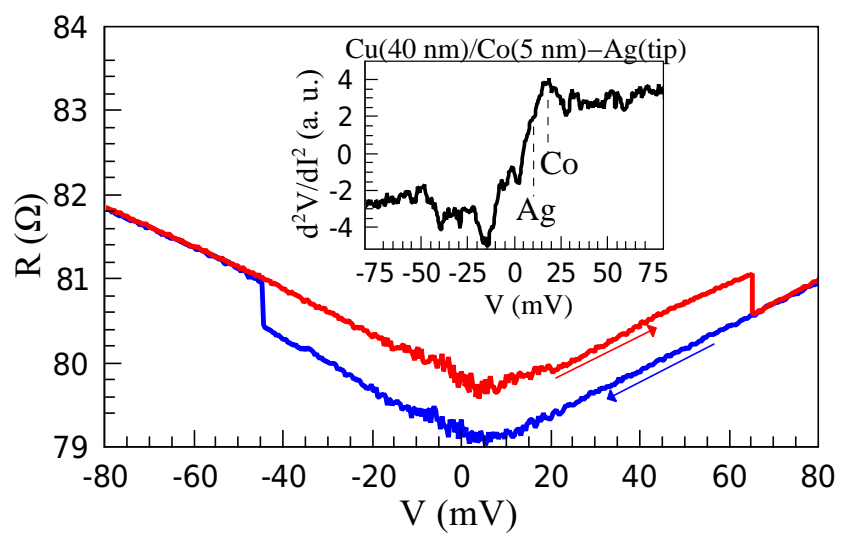

FIG. 2: Resistance $(R=V / I)$ and PC spectrum $\left(d^{2} V / d I^{2}\right.$; inset) of a $\mathrm{Co}(5 \mathrm{~nm})-\mathrm{Ag}($ tip $) \mathrm{PC}$ with $R_{\mathrm{PC}}=80 \Omega$ and $r_{\mathrm{PC}} \approx 1.5 \mathrm{~nm}$. Vertical dashed lines in the inset show the positions of the main phonon maxima in $\mathrm{PC}$ spectra of $\mathrm{Ag}$ and Co [13].

as described in [6, 7]. Fig. 2 shows the data for one of our smallest PC's, with the resistance of about $80 \Omega$ and the radius of the contact core estimated at $1.5 \mathrm{~nm}$ using the well-known Sharvin formula. The PC resistance shows pronounced hysteresis, which has two distinct stable states at zero bias and appears indistinguishable from the STT hysteresis frequently reported for three-layer spin-valves [12].

The data for our nanometer scale PCs, of the kind shown in Fig. 2, allow us to draw the following important conclusions. First, the presence of the Co 23] phonon maximum indicates that the $\mathrm{PC}$ consists of the ferromagnetic metal of good crystalline quality. Secondly, the rather high resistance of this $\mathrm{PC}$ means that the size of the contact core is in fact smaller than the exchange length in Co $\left(l_{\mathrm{ex}} \approx 4-5 \mathrm{~nm}\right)$, and much smaller than any domain wall that can be created in the ferromagnet (domain wall length $l_{\text {Dw }}$ typically $5-10$ times the exchange length [14]). Fig. 1(b) illustrates these fundamental ferromagnetic length scales in comparison to our experimental results. In the case of our thinner $5 \mathrm{~nm}-\mathrm{Co}$ film (Fig. 2) the thickness is approximately the same as the bulk exchange length in Co, so any volume-like domain walls along the current can be excluded. In the case of our thicker ferromagnetic films (100 nm thick Co, Fig. 3), possible domain walls would be far outside the PC core and thus make essentially no contribution to the measured resistance. Based on this principal comparisons we can rule out the interpretation of the resistance hysteresis as due to a bulk-like domain-wall magnetoresistance (MR) [15], and conclude that the observed STT switching must be due to the spins in the surface layer of the ferromagnet, changing their orientation with respect to the interior spins [7].

In order to make such surface versus bulk spin re-

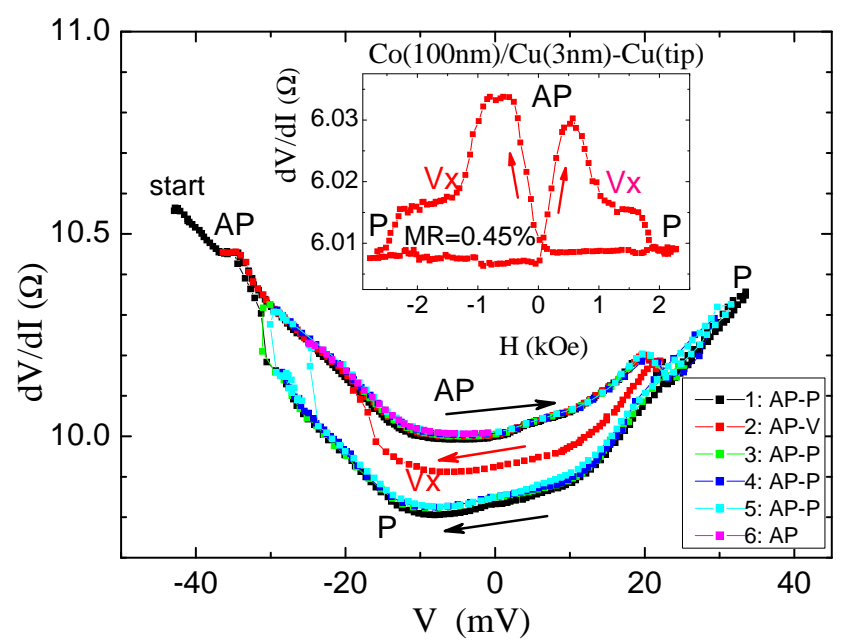

FIG. 3: (Color online) Differential resistance $d V / d I$ of a $\mathrm{Co}(100 \mathrm{~nm}) / \mathrm{Cu}(3 \mathrm{~nm}$ cap $)-\mathrm{Cu}($ tip $)$ contact vs bias voltage, showing six sequentially recorded $d V / d I(V)$ sweeps. In addition to the high- $\mathrm{R}$ and low- $\mathrm{R}$ states, corresponding to the anti-parallel (AP) and parallel (P) states of the surface spinvalve, a stable intermediate resistance state $(\mathrm{Vx})$ is produced in one of the sweeps denoted as a vortex state (red curve). The arrows indicate the voltage sweep direction. $T=4.2 \mathrm{~K}$, $H=0$. Inset: $d V / d I$ of another PC vs magnetic field at $I_{\mathrm{PC}}=16 \mu \mathrm{A}$, showing three stable resistance states labeled as $\mathrm{AP}, \mathrm{P}$, and $\mathrm{Vx}$, in analogy to $\mathrm{PC}$ in the main panel. The $\mathrm{P}-\mathrm{AP}$ and $\mathrm{P}-\mathrm{Vx} \mathrm{MR}$ vs field is the same in magnitude as the STT MR steps measured in $d V / d I(V)$ (not shown). $T=4.2 \mathrm{~K}$.

orientations possible either the exchange interaction or anisotropy, or both must be of different strength at the interface compared to the interior of the ferromagnet [10, 11]. The data and the micromagnetic simulations below demonstrate these surface magnetism effects in the current and field driven $\mathrm{MR}$ of $\mathrm{N} / \mathrm{F}$ interfaces.

Fig. 3 shows resistance versus bias voltage data for a $\sim 10 \Omega \mathrm{Co}-\mathrm{Cu}$ PC. Five out of six $d V / d I(V)$ sweeps recorded follow the major hysteresis loop, designated as the $\mathrm{P}$-to-AP switching loop. This current driven $\mathrm{P}-\mathrm{AP}$ MR of approximately $1.8 \%$ is essentially the same as the field driven $\mathrm{P}-\mathrm{AP} \mathrm{MR}$ of $1.6 \%$ (not shown), which shows the high reproducibility of the micromagnetic states involved. One sweep (No.2, red curve) shows a third resistance state, found at the mid-point between the $\mathrm{P}$ and AP resistances. This intermediate resistance position means that effectively only one half of the surface spins participating in the magneto-transport are in the AP state - the configuration expected for a spin vortex state. Indeed, if the spins at the interface, within the contact core, could form a stable vortex, the MR of the interface should have form shown in Fig. 3, with three approximately equidistant levels.

Of the PCs showing 3-level hysteresis, which is a $10 \%$ subset of the PC's showing hysteretic $d V / d I(V, H)$, some 


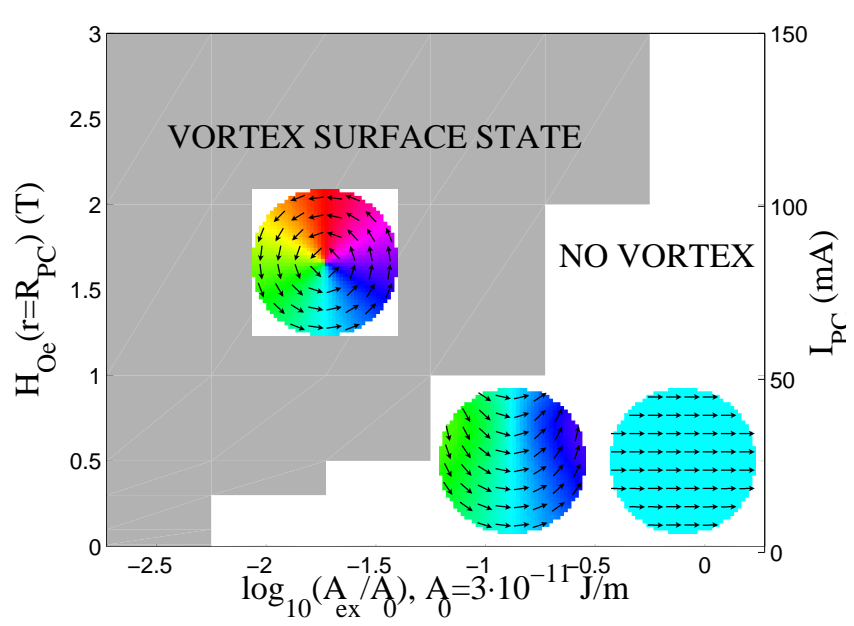

FIG. 4: (Color online) Vortex stability diagram (gray/white) as a function of the exchange stiffness and the Oersted field ( $\left.H_{\mathrm{Oe}}\right)$ of the bias current. Here $H_{\mathrm{Oe}}$ is the minimum field required for nucleating a stable vortex in a $1 \mathrm{~nm}$ thin disk of $R_{\mathrm{PC}}=10 \mathrm{~nm}$. $H_{\mathrm{Oe}}$ is zero at $r=0$ and maximum at $r=R_{\mathrm{PC}}$.

contacts display a very characteristic field dependence of MR 24]. An example of this is shown in the inset to Fig. 3 for another $\mathrm{Co}-\mathrm{Cu}$ contact. Sweeping the external field from the saturated $\mathrm{P}$ state of the $\mathrm{PC}$ through zero induces a switching in the softer underlying Co film with respect to the surface magnetic layer, 25] thereby placing the interface/bulk into the AP state. As the field is increased further, the intermediate-resistance state appears at $1-2 \mathrm{kOe}$, and is subsequently saturated in still higher fields. The shape of the $d V / d I(V, H)$ curves has a striking similarity to the three-level hysteresis recently reported in 17] (see their Figs. 2,3), where a spin-vortex state was intentionally created in one of the ferromagnetic layers (ring-shaped in fabrication) of a $100 \mathrm{~nm}$ scale spin-valve nanopillar. Similar to [17], we can transform our $d V / d I(H)$ 2-level $\mathrm{P}$-AP hysteresis loops into 3-level $\mathrm{P}-\mathrm{V}-\mathrm{AP}$ loops by suitably limiting the field sweep amplitude. The same three-level hysteresis is found also in zero field $d V / d I(V)$ sweeps for this contact, with the same magnitude $(\sim 0.5 \%)$ of the $\mathrm{P}-\mathrm{AP}$ MR (not shown). These vortex-no vortex transformations are fully reversible. The high similarity between our three-fold hysteresis data and the vortex-based spin-valves of [17] provides further support for our interpretation of the observed switching behavior in nano-sized PCs in terms of surface spin-valve and spin-vortex states, which can be reoriented by current-induced STT or external magnetic field.

It is informative to note that non-uniform magnetization states, such as magnetic vortices, were suggested to explain spin dynamics in larger, $100 \mathrm{~nm}$ scale point contacts [18, 19], where the vortex core would oscil- late at relatively low-frequency $(<500 \mathrm{MHz})$, driven by a spin-polarized current. In [20] we observed a peak in $d V / d I(V)$ for $10 \mathrm{~nm}$ scale $\mathrm{Co}-\mathrm{Cu}$ point contacts stimulated by external RF fields, which we interpreted as excitations of resonant magnetization precession. The typical resonant frequencies observed in our experiments were in the range 1-10 GHz, which is relatively low compared to the FMR frequency expected for a uniformly magnetized film or particle in a field of about $1 \mathrm{~T}(>10 \mathrm{GHz})$. Therefore, we cannot exclude that the spin-torque dynamics effects we observed previously originate from spin dynamics in non-uniformly magnetized nano-objects, such as spin vortices, where the characteristic frequencies are typically lower than those for uniform spin systems.

The fact that the nanopillars of [17] and our PCs differ in size by an order of magnitude is important. In our case, in order to produce the relatively large MR observed, the spin-vortex must be of the similar small size as the contact core. Our numerical micromagnetic analysis 21] shows that such $10 \mathrm{~nm}$ scale spin-vortex states can only be produced if the exchange interaction strength is assumed to be significantly lower at the interface compared to that in the bulk. To illustrate this, we simulate stable spin configurations in a $1 \mathrm{~nm}$ thin disk of radius $10 \mathrm{~nm}$. The disk is discretized into a 3D mesh of $0.5 \times 0.5 \times 0.5 \mathrm{~nm}^{3}$ cubic cells having the magnetization and anisotropy typical for Co, and varying exchange stiffness $A$ from $10^{-2} A_{0}$ to the bulk value of $A_{0}=3 \cdot 10^{-11} \mathrm{~J} / \mathrm{m}$. We assume that the bias current $I$ is uniformly distributed across the PC, and the Oersted field it produces within the contact core to be $H_{\mathrm{Oe}}(r)=H_{0}\left(r / R_{\mathrm{PC}}\right)$, where $H_{0}=\mu_{0} I /\left(2 \pi R_{\mathrm{PC}}\right)$. The resulting vortex phase diagram obtained by the micromagnetic minimization [16] is shown in Fig. 4. The results of this qualitative simulation are quite intuitive no nano-sized vortex states can be formed unless the exchange is allowed to decrease substantially, and that the Oersted field of the driving current strongly promotes the vortex state.

To further investigate the vortex stability in the PC we have determined the vortex nucleation probability from micromagnetic simulations where in each run the initial magnetization randomized and subsequently equilibrated. The results of these simulations for $1 \mathrm{~nm}$ thin disks of $10 \mathrm{~nm}$ and $25 \mathrm{~nm}$ in radius are shown in Fig. 5 . The probability for each data point is an average of 20 runs and is a function of the relative exchange stiffness $A / A_{0}$ in the ferromagnet. The following material parameters were used: saturation magnetization $M s=1.25 \cdot 10^{6}$ A/m, anisotropy energy density $K_{u}^{O P}=10^{5} \mathrm{~J} / \mathrm{m}^{3}$, micromagnetic mesh size of $1 \mathrm{~nm}$. The value of the out-of-plain uniaxial anisotropy $K_{u}^{O P}$ does not change the probability significantly. The out-of-plain nature of this anisotropy can originate due to mechanical stress in the contact core, discussed in detail previously 22]. This simulation indicates that the experimentally observed fraction of the 


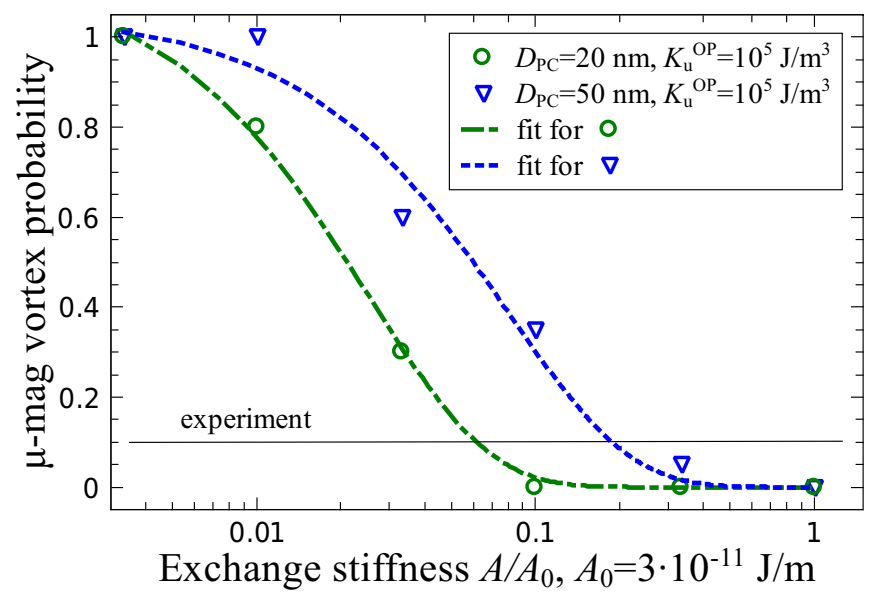

FIG. 5: (Color online) Vortex nucleation probability as a function of the exchange stiffness constant for $1 \mathrm{~nm}$ thin, $20(10) \mathrm{nm}$ and 50(25) nm diameter(radius) disks at zero external field and bias current. For comparison, the horizontal line shows the experimentally measured probability of observing PCs with tri-stable hysteresis.

vortex-like PCs of $10 \%$ (black horizontal line in Fig. 5.) corresponds to a reduction in the exchange stiffness in Co of approximately one order of magnitude (5-20\%, depending on the specific parameters chosen in the simulation).

We conclude that energetically distinct surface spin states play the key role in the STT effect in nano-scale magnetic PCs. These states can be uniform-spin or vortex-spin states at the surface or interface of the ferromagnet, and can be manipulated by combining the STT effects and the field of the driving current through the interface, as well as the externally applied field. Our results highlight the importance of the magnetic nature of the $\mathrm{N} / \mathrm{F}$ interface, and especially the strength of the interface versus bulk exchange interaction, for the spin-dependent transport on the nanoscale.

Acknowledgments. The support of FP7 programm of EU under project STEELE \#225955 and "HAHO"programm NAS of Ukraine under project \#02/09-H is acknowledged.

[1] L. Berger, Phys. Rev. B 54, 9353 (1996).

[2] J. Slonczewski, J. Magn. Magn. Mater. 159, L1 (1996).

[3] Y. Ji, C. L. Chen, and M. D. Stiles, Phys. Rev. Lett. 90, 106601 (2003).

[4] M. L. Polianski and P. W. Brouwer, Phys. Rev. Lett. 92, 026602 (2004).

[5] M. D. Stiles, J. Xiao, and A. Zangwill, Phys. Rev. B 69, 054408 (2004).

[6] I. K. Yanson, Yu. G. Naidyuk, D. L. Bashlakov, V. V.
Fisun, O. P. Balkashin, V. Korenivski, A. Konovalenko, and R. I. Shekhter, Phys. Rev. Lett. 95, 186602 (2005).

[7] I. K. Yanson, Yu. G. Naidyuk, V. V. Fisun, A. Konovalenko, O. P. Balkashin, L. Yu. Triputen, and V. Korenivski, Nano Letters, 7, 927 (2007).

[8] D. C. Ralph, M. D. Stiles, J. Magn. Magn. Mater. 320, 1190 (2008).

[9] J. C. Slonczewski, J. Magn. Magn. Mater. 195, L261 (1999).

[10] M. Gruyters, T. Bernhard, H. Winter, Phys. Rev. Lett. 94, 227205 (2005).

[11] H. B. Zhao, D. Talbayev, G. Lpke, A. T. Hanbicki, C. H. Li, and B. T. Jonker, Appl. Phys. Lett. 91, 052111 (2007).

[12] F. J. Albert, N. C. Emley, E. B. Myers, D. C. Ralph, and R. A. Buhrman, Phys. Rev. Lett. 89, 226802 (2002).

[13] Yu. G. Naidyuk and I. K. Yanson, Point-Contact Spectroscopy, Springer Series in Solid-State Sciences, Vol.145 (Springer Science+Business Media, Inc, 2005).

[14] W. Rave, K. Fabian, and A. Hubert, J. Magn. Magn. Mater. 190, 332 (1998).

[15] T. Y. Chen, Y. Ji, C. L. Chien and M. D. Stiles, Phys. Rev. Lett. 93, 026601 (2004).

[16] M. J. Donahue and D. G. Porter, OOMMF User's Guide, ver. 1.0, (1999); http://math.nist.gov/oommf

[17] T. Yang, M. Hara, A. Hirohata, T. Kimura, and Y. Otani, Appl. Phys. Lett. 90, 022504 (2007).

[18] M. R. Pufall, W. H. Rippard, M. L. Schneider, and S. E. Russek, Phys. Rev. B 75, 140404 (2007).

[19] Q. Mistral, M. van Kampen, G. Hrkac, Joo-Von Kim, T. Devolder, P. Crozat, C. Chappert, L. Lagae, and T. Schref, Phys. Rev. Lett. 100, 257201 (2008).

[20] O. P. Balkashin, V. V. Fisun, I. K. Yanson, L. Yu. Triputen, A. Konovalenko, and V. Korenivski, Phys. Rev. B 79, 092419 (2009).

[21] Details to be published elsewhere; see also K. Yu. Guslienko and K. L. Metlov, Phys. Rev. B 63, 100403 (2001); K. L. Metlov and K. Yu. Guslienko, J. Magn. Magn. Mater. 242-245 1015 (2002).

[22] A. Konovalenko, V. Korenivski, I. K. Yanson, Yu. G. Naidyuk, V. V. Fisun, O. P. Balkashin, and L. Yu. Triputen, J. Appl. Phys. 101, 09A513 (2007)

[23] The poorly resolved Ag phonon maximum in Fig. 2(inset) is due to a few times weaker electron-phonon interaction in Ag compared to that in Co (see, e. g., [13]), and possibly due to a smaller partial-volume of the PC core occupied by Ag.

[24] The correlation between the field and current induced magneto-resistance is normally taken as a confirmation of an STT effect (see e. g. [12]). Obviously, a homogeneous external magnetic field does not favor spin vortex states, which makes them much less probable than the uniform spin states.

[25] According to [10]:" For pure Co film the reversal of the bulk magnetization is preceded by a complete reversal of the surface magnetization", however Fig. 2e from this paper displays that in the case of the less perfect Co film noticeable part of surface requires higher than for a bulk field for a complete reversal. In our case the surface layer under study is restricted within a nanoscale PC size, therefore it is pinned strongly compared to the bulk and needs higher field for reversal of magnetization. 